\title{
Determination of Sterane and Triterpane in the Tamsagbulag Oilfield
}

\author{
Erdenee Enkhtsetseg ${ }^{1}$, Batdelger Byambagar ${ }^{1}$, Dalantai Monkhoobor ${ }^{2}$, \\ Budeebazar Avid $^{3 *}$, Avirmed Tuvshinjargal ${ }^{4}$ \\ ${ }^{1}$ Mongolian University of Science and Technololgy, Ulaanbaatar, Mongolia \\ ${ }^{2}$ National University of Mongolia, Ulaanbaatar, Mongolia \\ ${ }^{3}$ Mongolian Academy of Sciences, Ulaanbaatar, Mongolia \\ ${ }^{4}$ Petroleum authority of Mongolia, Ulaanbaatar, Mongolia \\ E-mail: *avidmas@gmail.com
}

Received March 9, 2011; revised April 15, 2011; accepted April 26, 2011

\begin{abstract}
Crude oils representing four different holes from the Tamsagbulag oilfield were analyzed by gas chromatography-mass spectrometry (GC/MS) spectroscopy. 52 biomarker compounds, 34 pentacyclic triterpanes and 18 steranes were identified and semi-quantitatively determined by selective ion monitoring (SIM) chromatography. Depending on the oil source, the variation in the pentacyclic triterpanes distribution is more significant than the variation in the sterane distribution. It is said that pregnane and honopregnane originated during the period of very salty sedimentary accumulation in the condition of diagenesis.
\end{abstract}

Keywords: Crude Oil, Biomarker, GC-MS, Tamsagbulag Oilfield

\section{Introduction}

Biomarkers are complex molecular fossils derived from once living organisms and it can provide information on the organic source materials, environmental conditions during its deposition, the thermal maturity experienced by a rock or oil, and the degree of biodegradation. Studies on identification and quantification of biomarker compounds have obtained great importance for recognition and classification of crude oil source. Sterane and hopane biomarkers have great potential in helping to identify and allocate hydrocarbon sources [1-9].

It is very important to study polyaromatic aliphatic hydrocarbons with carbon of $\mathrm{C}_{27}$ or higher hydrocarbon chain like steroid and triterpenoid for the geochemical investigation of crude oil and other caustobiolithes. The chemical type and relative content of biomarker compounds in specific oil is considered as its unique fingerprint. Among the various types of biomarkers, triterpanes and steranes are the best target molecules for identification and quantification. These molecules, with a molecular weight of about 250 - 400, possess a relative high concentration in crude oil and they are resistant to petrochemical and microbial degradation [4,5]. Therefore, they have found their use in tracing the oil source, its characteristics, degree of weathering and the fate of spilled oil in the environment [6,7]. Utilizing the GC and GC/MS technique made it possible to arrive at a clear characterization and classification of crude oils according to their sources [8]. Source specific parameters (viz. DBT/P, Pr/Ph, C29/C27 sterane and hopane/sterane ratios and tri- and tetra-cyclic terpane distributions) indicate that family oils derived from a marine shale deposited under sub-oxic conditions, whereas family oils derived from terrigenous source, contain n-alkanes that are less depleted in ${ }^{13} \mathrm{C}$, consistent with their derivation from a terrigenous source rock and their higher thermal maturity. The more negative $\mathrm{n}$-alkane $\Omega{ }^{13} \mathrm{C}$ profiles of the family oils reflect their marine source [9]. The research demonstrated that the biomarker distribution and carbon isotopic composition could be used to differentiate oils derived from various lacustrine environments $[10,11]$.

Recently several researchers have paid more attention on biomarker and geochemical investigation of the Mongolian crude oil. Composition of the hydrocarbons and structure of the heteroatom compounds with high molecular weight from the Zuunbayan, Tsagaan els and 19-3, 19-12, 19-13, 19-14, 19-17 wells of Tamsagbulag oilfields have been studied by Khongorzul. Based on the results, the author has estimated product properties of 
crude oil fractions and proposed it's suitable applicaton and as well as refinery process [12]. Another investigation has been done on Toson Uul and Zuunbayan oil fields, specially it is focused on the effect of depths for the properties and geochemical study of crude oil and sedimentary rock [13]. The structure and compositions of the hydrocarbons from the Tamsagbulag and Zuunbayan oilfields are studied by Sainbayar [14]. The author has focused on the compositions of the light fractions, especially on normal and isoparapynic hydrocarbons and tried to characterize the geochemical classification.

The aim of this research is to study crude oils from four new holes from the Tamsagbulag oilfield by using chromatographic method.

\section{Materials and Methods}

The crude oil samples representing the 19-14, 19-28, 19-31 and 19-34 wells from the Tamsagbulag oilfield from Mongolia have been selected for investigation. The selected samples are separated into 3 different fractions like paraffin, naphtene and aromatics. GC/MS measurements were performed on a Thermo-Finnigan Trace DSQ mass spectrometer coupled to a Thermo Finnigan Trace GC with a split injector (1:20) and a $0.25 \mu \mathrm{m}$ HP-5MS fused silica capillary column with a $60 \mathrm{~m} \times 0.35 \mathrm{~mm}$ inner diameter. The temperature was held at $40^{\circ} \mathrm{C}$ for 3 min and then increased to $250^{\circ} \mathrm{C}$ at $5^{\circ} \mathrm{C} / \mathrm{min}$ intervals, with helium flow rate of $3 \mathrm{ml} / \mathrm{min}$. The EI-MS ionization voltage was $70 \mathrm{eV}$ (electron impact ionization), and the ion source and interface temperature were both $250^{\circ} \mathrm{C}$.

\section{Results and Discussion}

The two classes of biomaker, pentacyclic triterpanes and steranes were identified under the chosen MS condition. Together with steranes, terpanes, triterpanes belong to the most important petroleum hydrocarbons that retained the characteristic structure of the original biological compounds [2]. Tricyclics, tetracyclics, hopanes, and other compounds contribute to the sterane and terpane fingerprint mass chromatogram $(\mathrm{m} / \mathrm{z}=217$ and 191) commonly used to relate oils and source rocks. GC-MS chromatograms of the crude oil from Tamsagbulag oilfield are shown in Figures 1-2, the assignments of the peaks are summarized in Tables 1-2.

Molecular ratios measured from these mass chromatograms help illustrate similarities in the distributions of these biomarker compounds.

The crude oil was characterized by measuring the normalized peak heights of 18 steranes and 34 pentacyclic triterpanes.

The sterane and terpane concentrations of the crude oil from 19-14, 19-28, 19-31 and 19-34 wells of the Tamsagbulag oilfield are shown in Tables 3-4.

Based on the results shown in above, the contents of the biosteranes, isosteranes and diasteranes and composition of the hydrocarbons from the hopane homologies were incorporated in Tables $\mathbf{5}$ and $\mathbf{6}$.

The tables show that the composition of petroleum from some holes of Tamsagbulag oil field contains biosteranes and isosteranes with hydrocarbon atoms of $\mathrm{C}_{27}$ $\mathrm{C}_{29}$ and the ethylcholestanes with hydrocarbon atoms of $\mathrm{C}_{29}$ make up much of it. It means that major organic substances of this petroleum originated from oddments of upper plants on the Earth (Figure 1, Tables 3 and 5.)

Therefore pregnane $\left(\mathrm{C}_{21}\right)$ and honopregnane $\left(\mathrm{C}_{22}\right)$ which are the parts with small molecules of homologous alignment of sterane were found in the petroleum of the above mentioned oil field. It is said that pregnane and

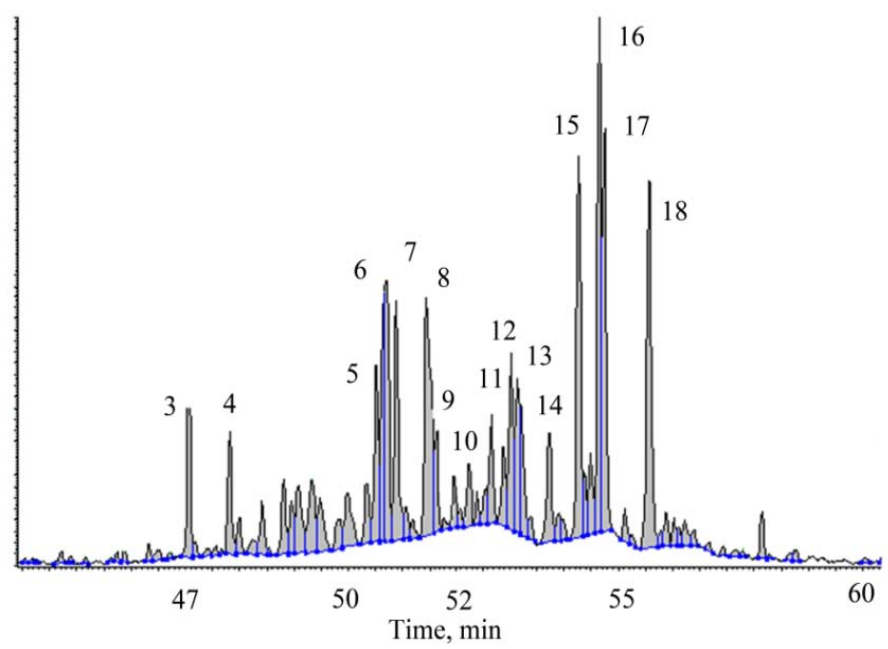

Figure 1. Mass chromatograms of oil samples of 19-31 well of the Tamsagbulag oilfield for steranes (m/z 217). 


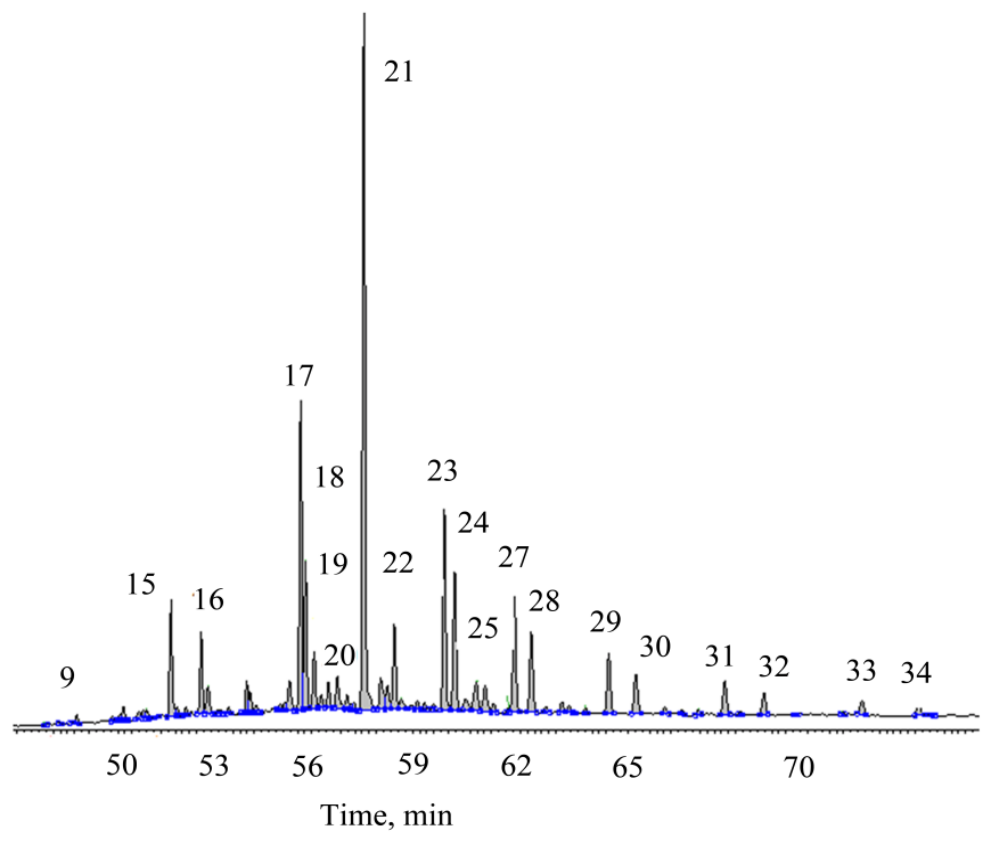

Figure 2. Mass chromatograms of oil samples of 19-31 well of the Tamsagbulag oilfield for terpanes (m/z 191).

Table 1. GC-MS peak identification for Figure 1.

\begin{tabular}{clc}
\hline Peak label & \multicolumn{1}{c}{ Compound } & Elemental composition \\
\hline 3 & 20S-áâá-Diacholestane & $\mathrm{C}_{27} \mathrm{H}_{48}$ \\
4 & 20R-áâá-Diacholestane & $\mathrm{C}_{27} \mathrm{H}_{48}$ \\
5 & 20S-ááá-Cholestane & $\mathrm{C}_{27} \mathrm{H}_{48}$ \\
6 & 20R-áââ-Cholestane & $\mathrm{C}_{27} \mathrm{H}_{48}$ \\
7 & 20S-áââ-Cholestane & $\mathrm{C}_{27} \mathrm{H}_{48}$ \\
8 & 20R-ááá-Cholestane & $\mathrm{C}_{27} \mathrm{H}_{48}$ \\
9 & 20R-24-Ethyl-10á13â17á-diacholestane & $\mathrm{C}_{29} \mathrm{H}_{52}$ \\
10 & 20S-24-Ethyl-10á13á18â-diacholestane & $\mathrm{C}_{29} \mathrm{H}_{52}$ \\
11 & 20S-24-Methyl-ááá-cholestane & $\mathrm{C}_{28} \mathrm{H}_{50}$ \\
12 & 20R-24-Methyl-áââ-cholestane & $\mathrm{C}_{28} \mathrm{H}_{50}$ \\
13 & 20S-24-Methyl-áââ-cholestane & $\mathrm{C}_{28} \mathrm{H}_{50}$ \\
14 & 20R-24-Methyl-ááá-cholestane & $\mathrm{C}_{28} \mathrm{H}_{50}$ \\
16 & 20S-24-Ethyl-ááá-cholestane & $\mathrm{C}_{29} \mathrm{H}_{52}$ \\
17 & 20R-24-Ethyl-áââ-cholestane & $\mathrm{C}_{29} \mathrm{H}_{52}$ \\
18 & 20S-24-Ethyl-áââ-cholestane & $\mathrm{C}_{29} \mathrm{H}_{52}$ \\
$\mathrm{C}_{29} \mathrm{H}_{52}$ & \\
\hline
\end{tabular}

honopregnane originated during the period of very salty sedimentary accumulation in the condition of diagenesis. In the petroleum from holes 19-14, 19-28 and 19-31 of Tamsagbulag, most of diasteranes which are the newly formed isomer of biosteranes are hydrocarbon compounds of $\mathrm{C}_{27}$ (7.5\% - 9.5\% relatively). The petroleum of the hole 19-34 contains relatively little amount of hydrocarbon compounds of $\mathrm{C}_{27}$ which is only $1.09 \%$. In other words, $\mathrm{C}_{27}>\mathrm{C}_{29}$ for diasteranes of petroleum of holes 19-14, 19-28 and 19-31 while $\mathrm{C}_{27}<\mathrm{C}_{29}$ for hole 19-34. Diasteranes of petroleum are useful for determining component of sediment lithology comprised fundamental source and original substance of petroleum. The $\mathrm{C}_{29}$ norhopanes and $\mathrm{C}_{30}$ hopanes are the dominating biomarkers in all the crude oils which is characteristic for source rocks rich in terrestrial organic matter [15].

These hydrocarbons' amount in the petroleum of holes $19-14,19-28$ and 19-31 is relatively more than in petroleum of hole 19-34 so it means that the origin of the petroleum of these 3 three holes except 19-34 is relevant with sedimentary rocks which are rich in aluminum silicate. Tricyclicterpane amounted of 3\% - 15\% was discovered from among class of hydrocarbons of terpane and contents of hopane and norhopane in the 4 holes of the petroleum were $44 \%$ - 47.5\%, $6 \%$ - $8 \%$, respectively. Homohopanes amount in hydrocarbons of terpane in the petroleum of the aforementioned 4 holes was accounted for $25 \%$ - 35\%. There was a constant decrease of homohopanes with $\mathrm{C}_{32}-\mathrm{C}_{35}$ and each pair of epimers had plenty of (22-S)-epimers (Tables 2 and 4). Processing level of 
Table 2. GC-MS peak identification for Figure 2.

\begin{tabular}{|c|c|c|}
\hline Peak label & Compound & Elemental composition \\
\hline 1 & $\mathrm{C}_{19}, 14 \hat{a}$ (methyl)-Tricyclicterpane & $\mathrm{C}_{19} \mathrm{H}_{34}$ \\
\hline 2 & $\mathrm{C}_{20}, 13 \hat{a}(\mathrm{H}), 14 a ́(\mathrm{H})$-Tricyclicterpane & $\mathrm{C}_{20} \mathrm{H}_{36}$ \\
\hline 3 & $\mathrm{C}_{21}, 13 \hat{a}(\mathrm{H}), 14 a ́(\mathrm{H})$-Tricyclicterpane & $\mathrm{C}_{21} \mathrm{H}_{38}$ \\
\hline 4 & $\mathrm{C}_{22}, 13 \hat{a}(\mathrm{H}), 14 a ́(\mathrm{H})$ - Tricyclicterpane & $\mathrm{C}_{22} \mathrm{H}_{40}$ \\
\hline 5 & $\mathrm{C}_{23}, 13 \hat{a}(\mathrm{H}), 14 a ́(\mathrm{H})$-Tricyclicterpane & $\mathrm{C}_{23} \mathrm{H}_{42}$ \\
\hline 6 & $\mathrm{C}_{24}, 13 \hat{a}(\mathrm{H}), 14 a ́(\mathrm{H})$-Tricyclicterpane & $\mathrm{C}_{24} \mathrm{H}_{44}$ \\
\hline 7 & $\mathrm{C}_{25}, 13 \hat{a}(\mathrm{H}), 14 a ́(\mathrm{H})$-Tricyclicterpane & $\mathrm{C}_{25} \mathrm{H}_{46}$ \\
\hline 8 & $\mathrm{C}_{26}$, Tricyclicterpane & $\mathrm{C}_{26} \mathrm{H}_{48}$ \\
\hline 9 & $\mathrm{C}_{26}$, Tricyclicterpane & $\mathrm{C}_{26} \mathrm{H}_{48}$ \\
\hline 10 & $\mathrm{C}_{24}$, Tricyclicterpane & $\mathrm{C}_{24} \mathrm{H}_{44}$ \\
\hline 11 & $\mathrm{C}_{28}$, Tricyclicterpane & $\mathrm{C}_{28} \mathrm{H}_{50}$ \\
\hline 12 & $\mathrm{C}_{28}$, Tricyclicterpane & $\mathrm{C}_{28} \mathrm{H}_{50}$ \\
\hline 13 & $\mathrm{C}_{29}$, Tricyclicterpane & $\mathrm{C}_{29} \mathrm{H}_{52}$ \\
\hline 14 & $\mathrm{C}_{29}$, Tricyclicterpane & $\mathrm{C}_{29} \mathrm{H}_{52}$ \\
\hline 15 & 18á, -22, 29, 30-Trisnorhopane(Ts) & $\mathrm{C}_{27} \mathrm{H}_{46}$ \\
\hline 16 & 17á, -22, 29, 30-Trisnorhopane(Tm) & $\mathrm{C}_{27} \mathrm{H}_{46}$ \\
\hline 17 & 17á, 21â-30-Norhopane & $\mathrm{C}_{29} \mathrm{H}_{50}$ \\
\hline 18 & $\mathrm{C}_{29} \mathrm{Ts}$ & $\mathrm{C}_{29} \mathrm{H}_{50}$ \\
\hline 19 & 18á(H)-Hopane & $\mathrm{C}_{30} \mathrm{H}_{52}$ \\
\hline 20 & 17â(H), 21á(H)-Normoretane & $\mathrm{C}_{29} \mathrm{H}_{50}$ \\
\hline 21 & $\mathrm{C}_{30} 17 a ́(\mathrm{H}), 21 \hat{a}(\mathrm{H})$-Hopane & $\mathrm{C}_{30} \mathrm{H}_{52}$ \\
\hline 22 & 17â(H), 21á(H)-Moretane & $\mathrm{C}_{30} \mathrm{H}_{52}$ \\
\hline 23 & 22S-17á(H), 21â(H)-Homohopane & $\mathrm{C}_{31} \mathrm{H}_{54}$ \\
\hline 24 & $\mathrm{C}_{31} 22 \mathrm{R}-17 a ́(\mathrm{H}), 21 \hat{a}(\mathrm{H})$-Homohopane & $\mathrm{C}_{31} \mathrm{H}_{54}$ \\
\hline 25 & Gammacerane & $\mathrm{C}_{30} \mathrm{H}_{52}$ \\
\hline 26 & 22R-17â(H), 21á(H)-Homomoretane & $\mathrm{C}_{31} \mathrm{H}_{54}$ \\
\hline 27 & 22S-17á(H), 21â(H)-Dihomohopane & $\mathrm{C}_{32} \mathrm{H}_{56}$ \\
\hline 28 & 22R-17á(H), 21â(H)-Dihomohopane & $\mathrm{C}_{32} \mathrm{H}_{56}$ \\
\hline 29 & 22S-17á(H), 21â(H)-Trihomohopane & $\mathrm{C}_{33} \mathrm{H}_{58}$ \\
\hline 30 & 22R-17á(H), 21â(H)-Trihomohopane & $\mathrm{C}_{33} \mathrm{H}_{58}$ \\
\hline 31 & 22S-17á(H), 21â(H)-Tetrahomohopane & $\mathrm{C}_{34} \mathrm{H}_{60}$ \\
\hline 32 & 22R-17á(H), 21â(H)-Tetrahomohopane & $\mathrm{C}_{34} \mathrm{H}_{60}$ \\
\hline 33 & 22S-17á(H), 21â(H)-Pentahomohopane & $\mathrm{C}_{35} \mathrm{H}_{62}$ \\
\hline 34 & 22R-17á(H), 21â(H)-Pentahomohopane & $\mathrm{C}_{35} \mathrm{H}_{62}$ \\
\hline
\end{tabular}

Table 3. Sterane concentration in the crude oil from Tamsagbulag oilfield, wt $\%$.

\begin{tabular}{|c|c|c|c|c|c|c|c|}
\hline \multirow{2}{*}{ ID } & \multirow{2}{*}{ Compound } & \multirow{2}{*}{ Formula } & \multirow{2}{*}{$\begin{array}{c}\text { Molecular } \\
\text { weight }\end{array}$} & \multicolumn{4}{|c|}{ Oil wells } \\
\hline & & & & 14 & 28 & 31 & 34 \\
\hline S1 & 5á(H), 14â(H)-Pregnane & $\mathrm{C}_{21} \mathrm{H}_{36}$ & 288 & 2.30 & 2.80 & 2.45 & 1.62 \\
\hline $\mathrm{S} 2$ & C22 Honopregnane & $\mathrm{C}_{22} \mathrm{H}_{38}$ & 302 & 1.57 & 1.77 & 1.52 & 0.93 \\
\hline S3 & 20S-áâá-Diacholestane & $\mathrm{C}_{27} \mathrm{H}_{48}$ & 372 & 4.79 & 5.46 & 4.56 & 0.48 \\
\hline S4 & 20R-áâá-Diacholestane & $\mathrm{C}_{27} \mathrm{H}_{48}$ & 372 & 3.17 & 4.12 & 3.03 & 0.61 \\
\hline S11 & 20S-ááá-Cholestane & $\mathrm{C}_{27} \mathrm{H}_{48}$ & 372 & 5.05 & 4.48 & 4.69 & 6.64 \\
\hline $\mathrm{S} 12$ & 20R-áââ-Cholestane & $\mathrm{C}_{27} \mathrm{H}_{48}$ & 372 & 6.08 & 5.97 & 6.45 & 5.39 \\
\hline $\mathrm{S} 13$ & 20S-áââ-Cholestane & $\mathrm{C}_{27} \mathrm{H}_{48}$ & 372 & 6.28 & 7.07 & 6.07 & 5.49 \\
\hline S14 & 20R-ááá-Cholestane & $\mathrm{C}_{27} \mathrm{H}_{48}$ & 372 & 6.38 & 4.73 & 6.02 & 9.78 \\
\hline S15 & 20R-24-Ethyl-10á13â17á-diacholestane & $\mathrm{C}_{29} \mathrm{H}_{52}$ & 400 & 3.41 & 2.99 & 3.25 & 3.39 \\
\hline S16 & 20S-24-Ethyl-10á13á18â-diacholestane & $\mathrm{C}_{29} \mathrm{H}_{52}$ & 400 & 1.62 & 1.82 & 1.74 & 1.07 \\
\hline $\mathrm{S} 17$ & 20S-24-Methyl-ááá-cholestane & $\mathrm{C}_{28} \mathrm{H}_{50}$ & 386 & 2.88 & 3.71 & 3.12 & 2.56 \\
\hline S18 & 20R-24-Methyl-áââ-cholestane & $\mathrm{C}_{28} \mathrm{H}_{50}$ & 386 & 2.44 & 2.92 & 2.62 & 3.67 \\
\hline S19 & 20S-24-Methyl-áââ-cholestane & $\mathrm{C}_{28} \mathrm{H}_{50}$ & 386 & 4.83 & 4.99 & 4.96 & 5.96 \\
\hline $\mathrm{S} 20$ & 20R-24-Methyl-ááá-cholestane & $\mathrm{C}_{28} \mathrm{H}_{50}$ & 386 & 3.74 & 3.71 & 3.84 & 5.58 \\
\hline $\mathrm{S} 21$ & 20S-24-Ethyl-ááá-cholestane & $\mathrm{C}_{29} \mathrm{H}_{52}$ & 400 & 10.13 & 8.53 & 9.83 & 12.01 \\
\hline $\mathrm{S} 22$ & 20R-24-Ethyl-áââ-cholestane & $\mathrm{C}_{29} \mathrm{H}_{52}$ & 400 & 13.08 & 13.24 & 12.51 & 11.22 \\
\hline $\mathrm{S} 23$ & 20S-24-Ethyl-áââ-cholestane & $\mathrm{C}_{29} \mathrm{H}_{52}$ & 400 & 10.26 & 10.70 & 11.43 & 9.08 \\
\hline $\mathrm{S} 24$ & 20R-24-Ethyl-ááá-cholestane & $\mathrm{C}_{29} \mathrm{H}_{52}$ & 400 & 12.00 & 11.00 & 11.91 & 14.51 \\
\hline
\end{tabular}


Table 4. Terpane concentration in the crude oil from Tamsagbulag oilfield, wt \%.

\begin{tabular}{|c|c|c|c|c|c|c|c|}
\hline \multirow{2}{*}{ ID } & \multirow{2}{*}{ Compound } & \multirow{2}{*}{ Formula } & \multirow{2}{*}{$\begin{array}{c}\text { Molecular } \\
\text { weight }\end{array}$} & \multicolumn{4}{|c|}{ Oil wells } \\
\hline & & & & 14 & 28 & 31 & 34 \\
\hline $\mathrm{T}_{1}$ & C19, 14â(methyl)-Tricyclicterpane & $\mathrm{C}_{19} \mathrm{H}_{34}$ & 262 & 0.55 & 0.70 & 0.57 & 0.38 \\
\hline $\mathrm{T}_{2}$ & C20, 13â(H), 14á(H)-Tricyclicterpane & $\mathrm{C}_{20} \mathrm{H}_{36}$ & 276 & 0.45 & 0.56 & 0.44 & 1.88 \\
\hline $\mathrm{T}_{3}$ & C21, 13â(H), 14á(H)-Tricyclicterpane & $\mathrm{C}_{21} \mathrm{H}_{38}$ & 290 & 0.49 & 0.67 & 0.48 & 4.53 \\
\hline $\mathrm{T}_{4}$ & C22, 13â(H), 14á(H)-Tricyclicterpane & $\mathrm{C}_{22} \mathrm{H}_{40}$ & 304 & 0.09 & - & 0.10 & 0.42 \\
\hline $\mathrm{T}_{5}$ & C23, 13â(H), 14á(H)-Tricyclicterpane & $\mathrm{C}_{23} \mathrm{H}_{42}$ & 318 & 0.42 & 0.50 & 0.39 & 1.87 \\
\hline $\mathrm{T}_{6}$ & C24, 13â(H), 14á(H)-Tricyclicterpane & $\mathrm{C}_{24} \mathrm{H}_{44}$ & 332 & 0.20 & 0.27 & 0.23 & 1.03 \\
\hline $\mathrm{T}_{7}$ & C25, 13â(H), 14á(H)-Tricyclicterpane & $\mathrm{C}_{25} \mathrm{H}_{46}$ & 346 & 0.10 & - & - & 0.81 \\
\hline $\mathrm{T}_{9}$ & C26, Tricyclicterpane & $\mathrm{C}_{26} \mathrm{H}_{48}$ & 360 & 0.15 & 0.16 & 0.14 & 0.53 \\
\hline $\mathrm{T}_{10}$ & C26, Tricyclicterpane & $\mathrm{C}_{26} \mathrm{H}_{48}$ & 360 & - & - & - & - \\
\hline $\mathrm{T}_{8}$ & C24, Tricyclicterpane & $\mathrm{C}_{24} \mathrm{H}_{44}$ & 332 & 1.19 & 1.29 & 1.14 & 1.52 \\
\hline $\mathrm{T}_{11}$ & C28, Tricyclicterpane & $\mathrm{C}_{28} \mathrm{H}_{50}$ & 388 & - & - & - & 1.17 \\
\hline $\mathrm{T}_{12}$ & C28, Tricyclicterpane & $\mathrm{C}_{28} \mathrm{H}_{50}$ & 388 & - & & - & 0.50 \\
\hline $\mathrm{T}_{13}$ & C29, Tricyclicterpane & $\mathrm{C}_{29} \mathrm{H}_{52}$ & 402 & - & - & - & 0.35 \\
\hline $\mathrm{T}_{14}$ & C29, Tricyclicterpane & $\mathrm{C}_{29} \mathrm{H}_{52}$ & 402 & - & - & - & 0.28 \\
\hline $\mathrm{T}_{15}$ & 18á, -22,29,30-Trisnorhopane(Ts) & $\mathrm{C}_{27} \mathrm{H}_{46}$ & 370 & 4.97 & 5.42 & 4.37 & 2.57 \\
\hline $\mathrm{T}_{16}$ & 17á, -22,29,30-Trisnorhopane(Tm) & $\mathrm{C}_{27} \mathrm{H}_{46}$ & 370 & 3.17 & 2.86 & 2.97 & 3.76 \\
\hline $\mathrm{T}_{19}$ & 17á, 21â-30-Norhopane & $\mathrm{C}_{29} \mathrm{H}_{50}$ & 398 & 11.77 & 9.97 & 11.44 & 13.49 \\
\hline $\mathrm{T}_{20}$ & C29Ts & $\mathrm{C}_{29} \mathrm{H}_{50}$ & 398 & 5.52 & 6.00 & 5.53 & 2.57 \\
\hline $\mathrm{T}_{21}$ & 18á(H)-Hopane & $\mathrm{C}_{30} \mathrm{H}_{52}$ & 412 & 2.72 & 3.26 & 2.50 & 1.02 \\
\hline $\mathrm{T}_{22}$ & 17â(H), 21á(H)-Normoretane & $\mathrm{C}_{29} \mathrm{H}_{50}$ & 398 & 1.19 & 1.47 & 1.08 & 0.60 \\
\hline $\mathrm{T}_{23}$ & 17á(H), 21â(H)-Hopane & $\mathrm{C}_{30} \mathrm{H}_{52}$ & 412 & 27.42 & 26.41 & 27.11 & 26.91 \\
\hline $\mathrm{T}_{24}$ & 17â(H), 21á(H)-Moretane & $\mathrm{C}_{30} \mathrm{H}_{52}$ & 412 & 3.51 & 3.48 & 3.62 & 3.53 \\
\hline $\mathrm{T}_{25}$ & 22S-17á(H), 21â(H)-Homohopane & $\mathrm{C}_{31} \mathrm{H}_{54}$ & 426 & 7.92 & 7.55 & 8.20 & 6.48 \\
\hline $\mathrm{T}_{26}$ & 22R-17á(H), 21â(H)-Homohopane & $\mathrm{C}_{31} \mathrm{H}_{54}$ & 426 & 6.08 & 6.01 & 6.20 & 5.02 \\
\hline $\mathrm{T}_{27}$ & Gammacerane & $\mathrm{C}_{30} \mathrm{H}_{52}$ & 412 & 1.63 & 1.72 & 1.89 & 3.28 \\
\hline $\mathrm{T}_{28}$ & 22R-17â(H), 21á(H)-Homomoretane & $\mathrm{C}_{31} \mathrm{H}_{54}$ & 426 & 1.22 & 1.14 & 1.63 & 2.15 \\
\hline $\mathrm{T}_{29}$ & 22S-17á(H), 21â(H)-Dihomohopane & $\mathrm{C}_{32} \mathrm{H}_{56}$ & 440 & 5.29 & 5.34 & 5.36 & 5.25 \\
\hline $\mathrm{T}_{30}$ & 22R-17á(H), 21â(H)-Dihomohopane & $\mathrm{C}_{32} \mathrm{H}_{56}$ & 440 & 3.82 & 4.13 & 3.89 & 2.75 \\
\hline $\mathrm{T}_{31}$ & 22S-17á(H), 21â(H)-Trihomohopane & $\mathrm{C}_{33} \mathrm{H}_{58}$ & 454 & 3.05 & 3.18 & 3.12 & 2.04 \\
\hline $\mathrm{T}_{32}$ & 22R-17á(H), 21â(H)-Trihomohopane & $\mathrm{C}_{33} \mathrm{H}_{58}$ & 454 & 2.10 & 2.45 & 2.29 & 1.19 \\
\hline $\mathrm{T}_{33}$ & 22S-17á(H), 21â(H)-Tetrahomohopane & $\mathrm{C}_{34} \mathrm{H}_{60}$ & 468 & 2.03 & 2.10 & 2.02 & 0.94 \\
\hline $\mathrm{T}_{34}$ & 22R-17á(H), 21â(H)-Tetrahomohopane & $\mathrm{C}_{34} \mathrm{H}_{60}$ & 468 & 1.29 & 1.39 & 1.34 & 0.57 \\
\hline $\mathrm{T}_{35}$ & 22S-17á(H), 21â(H)-Pentahomohopane & $\mathrm{C}_{35} \mathrm{H}_{62}$ & 482 & 0.98 & 1.11 & 1.12 & 0.40 \\
\hline $\mathrm{T}_{36}$ & 22R-17á(H), 21â(H)-Pentahomohopane & $\mathrm{C}_{35} \mathrm{H}_{62}$ & 482 & 0.70 & 0.87 & 0.81 & 0.21 \\
\hline
\end{tabular}

Table 5. The sterane concentration in the crude oil from some wells of the Tamsagbulag oilfield, wt \%.

\begin{tabular}{|c|c|c|c|c|}
\hline \multirow{2}{*}{ Compound } & \multicolumn{4}{|c|}{ Oil wells } \\
\hline & 14 & 28 & 31 & 34 \\
\hline 5á(H), 14â(H)-Pregnane & 2.30 & 2.80 & 2.45 & 1.62 \\
\hline C22 Honopregnane & 1.57 & 1.77 & 1.52 & 0.93 \\
\hline $\mathrm{C}_{27}$ & 11.43 & 9.21 & 10.71 & 16.42 \\
\hline $\mathrm{C}_{28}$ & 6.62 & 7.42 & 6.96 & 8.14 \\
\hline $\mathrm{C}_{29}$ & 22.13 & 19.53 & 21.74 & 26.52 \\
\hline Total biosterane & 40.18 & 36.16 & 39.41 & 51.08 \\
\hline $\mathrm{C}_{27}$ & 12.36 & 13.04 & 12.52 & 10.88 \\
\hline $\mathrm{C}_{28}$ & 7.27 & 7.91 & 7.58 & 9.63 \\
\hline $\mathrm{C}_{29}$ & 23.34 & 23.94 & 23.94 & 20.3 \\
\hline Total isosterane & 42.97 & 44.89 & 44.04 & 40.81 \\
\hline Total normal sterane & 83.15 & 81.05 & 83.45 & 91.89 \\
\hline $\mathrm{C}_{27}$ & 7.96 & 9.58 & 7.59 & 1.09 \\
\hline $\mathrm{C}_{28}$ & - & - & - & - \\
\hline $\mathrm{C}_{29}$ & 5.03 & 4.81 & 4.99 & 4.46 \\
\hline ¿sterane & 12.99 & 14.39 & 12.58 & 5.55 \\
\hline
\end{tabular}

the petroleum of these 4 holes was considered as low because none of diahopanes was found in their component. Some gammaceranes $(1.5 \%-3 \%)$ were found in the petroleum of holes 19-14, 19-28, 19-31 and 19-34 of Tamsagbulag oil field and this evidence shows characteristic of highly marine depositional conditions in the environment of ocean, sea and marshy lake.

\section{Conclusions}

1) The composition of the sterane and terpane hyrdocarbon derivatives of the Tamsagbulag crude oil well No. 14-19, 28, 31 and 34 has been determined by GC-MS.

2) Based on the contained biosteranes and isosteranes, 
Table 6. The hydrocarbon compositions of hopane derivatives in the crude oil from some wells of the Tamsagbulag oilfield, wt \%.

\begin{tabular}{|c|c|c|c|c|}
\hline \multirow{2}{*}{ Compound } & \multicolumn{4}{|c|}{ Oil wells } \\
\hline & 14 & 28 & 31 & 34 \\
\hline Tricyclicterpane & 3.64 & 4.15 & 3.49 & 15.27 \\
\hline Norhopane $\left(\mathrm{T}_{\mathrm{m}}+\mathrm{Ts}\right)$ & 8.14 & 8.28 & 7.34 & 6.33 \\
\hline Hopane & 47.43 & 45.64 & 46.58 & 43.99 \\
\hline Moretane & 4.7 & 4.95 & 4.7 & 4.13 \\
\hline Homohopane & 33.26 & 34.13 & 34.35 & 24.85 \\
\hline Homomoretane & 1.22 & 1.14 & 1.63 & 2.15 \\
\hline Gammacerane & 1.63 & 1.72 & 1.89 & 3.28 \\
\hline
\end{tabular}

the petroleum from some holes of Tamsagbulag oil field, originated from oddments of upper plants. The content of the gammacerane, pregnane and honopregnane found in the petroleum of the aforementioned oil field says that those are originated during the period of very salty sedimentary accumulation in the condition of diagenesis.

\section{References}

[1] G. D. Hobson and W. Pohl, "Modern Petroleum Technology,” Applied Science Publishers, London, 1973.

[2] J. M. Hunt, "Petroleum Geochemistry and Geology," Freeman Company, New York, 1979.

[3] J. G. Speight, "The Chemistry and Technology of Petroleum,” Marcel Dekker, Inc., New York, 1991.

[4] D. R. Luellen and D. Shea, "Semipermeable Membrane Devices Accumulate Conserved Rations of Sterane and Hopane Petroleum Biomarkers,” Chemosphere, Vol. 53, No. 7, 2003, pp. 705-713. doi:10.1016/S0045-6535(03)00576-9

[5] A. Hauser, H. Dashti and Z. H. Khan, "Identification of Biomarker Compounds in Selected Kuwait Crude Oils," Fuel, Vol. 78, No. 12, 1999, pp. 1483-1488. doi:10.1016/S0016-2361(99)00075-7

[6] J. Connan, J. Bouroullec, D. Dessort and P. Albrecht, "The Microbial Input in Carbonate-Anhydrite Facies of a Sabkha Palaeoenvironment from Guatemala: Molecular Approach,” Organic Geochemistry, Vol. 10, No. 1-3, 1986, pp. 29-50. doi:10.1016/0146-6380(86)90007-0

[7] A. A. Petrov, "Hydrocarbons of the Crude Oil," Nauka, Moscow, 1984.
[8] M. I. Roushdy, M. M. El Nady, Y. M. Mostafa, et al., "Biomarkers Characteristics of Crude Oils from Some Oilfields in the Gulf of Suez, Egypt," Journal of American Science, Vol. 6, No. 11, 2010, pp. 911-925.

[9] S. Aboglila, K. Grice, K. Trinajstic, et al., "Use of Biomarker Distributions and Compound Specific Isotopes of Carbon and Hydrogen to Delineate Hydrocarbon Characteristics in the East Sirte Basin (Libya)," Organic Geochemistry, Vol. 41, No. 12, 2010, pp. 1249-1258. doi:10.1016/j.orggeochem.2010.05.011

[10] R. P. Philp, J. G. Li and C. A. Lewis, “An Organic Geochemical Investigation of Crude Oils from Shanganning, Jianghan, Chaidamu and Zhungeer Basins, China,” Organic Geochemistry, Vol. 14, No. 4, 1989, pp. 447-460. doi:10.1016/0146-6380(89)90010-7

[11] R. P. Philp, R. T. Simoneit and T. D. Gilbert, "Advances in Organic Geochemistry,” Wiley, Chichester, 1981, pp. 698-704.

[12] B. Khongorzul, "Features of the Hydrocarbon Composition and High Molecular Compounds of the High Paraffinic Oil from Mongolia,” Ph.D. Thesis, Tomsk, 2008, pp. 67-77.

[13] V. Alimaa, "Effect of the Depths on Crude Oil Properties," Ph.D. Thesis, Mongolian University of Science and Tech- nology, UB, 2008, p. 118.

[14] A. Sainbayar, "Study on Hydrocarbon's Composition of Mongolian Petroleum,” Ph.D. Thesis, National University of Mongolia, Ulaanbaatar, 2006, p. 200.

[15] G. Demaison, A. J. Holck, R. W. Jones and G. T. Moore, "Predictive Source Bed Stratigraphy: A Guide to Regional Petroleum Occurrence,” Proceedings of the 11th World Petroleum Congress 2, PDI, Wiley, New York, 1984, pp. 17-29. 